((POLYENG.2020.0068.R1-accepted/edits))

\title{
Green composites based on Atriplex halimus fibers and PLA matrix
}

Hayet Latifa Boudjema ${ }^{1,2, *}$, Hayet Bendaikha ${ }^{2}$ and Ulrich Maschke ${ }^{3}$

1Université des Sciences et de la Technologie d'Oran—Mohamed Boudiaf, El Mnaouar, BP 1505, Bir El Djir 31000 Oran, Algeria

2 Laboratoire d'Ingénierie de la Sécurité Industrielle et du Développement Durable, Institut de Maintenance et de Sécurité Industrielle, Département de Sécurité Industrielle et Environnement, Université d'Oran 2 Mohamed Ben Ahmed, 31000 Oran, Algeria

3 Unité Matériaux et Transformations-UMET (UMR CNRS Nº 8207), Bâtiment C6, Université Lille 1—Sciences et Technologies, 59655 Villeneuve d'Ascq Cedex, France

*Corresponding author: e-mail: hayet.hayouta1331@gmail.com 
Abstract: This work focuses on the potential use of cellulose fibers extracted from Mediterranean saltbush (Atriplex halimus) as a filler in polymeric matrix.The fully biodegradable composites were prepared from PLA as matrix and micro-cellulose fibers ranging from 0 to $15 \mathrm{wt} \%$. The influence of the fiber content on the structure, mechanical, thermal and water absorption properties was evaluated. Mechanical results indicated that fibers acted effectively as reinforcement, increasing the tensile strength and the Young's modulus of PLA by $25 \%$ and $45 \%$, respectively. This is due to the good stress transfer betwen fibers and matrix through the strong interactions that have been evidenced by FTIR spectroscopy. The thermogravimetric analysis showed that PLA composites have a slightly lower degradation temperatures than the pure PLA but they still have favourable thermal stability. Water absorption measurements and biodegradability tests showed that the addidition addition of fibers accelerates degradation kineticks and confirm that the prepared composites are an environmentally safe material suited for different applications.

Keywords: biodegradability; cellulose fibers; mechanical properties; PLA polymer; thermal properties.

\section{Introduction}

The uncertainty of petroleum supply and environmental pollutions caused by nondegradable plastics have attracted world concerns over developing environmentally friendly materials from natural and renewable resources [1]

Polylactic acid is one of the most studied biodegradable polymers mainly because of its relatively high melting point, crystallinity and stiffness compared to other biopolymers. Currently, the majority of this linear aliphatic thermoplastic polyester is produced by polymerization of lactide, a cyclic dimer derived from lactic acid, which in turn is obtained by the fermentation of agricultural products such as corn, potato, cane sugar, and rice. In spite of its high biocompatibility and biodegradability, which make it a very good candidate for biomedical and packaging uses, a large number of its applications is still limited by its high production cost and brittleness [2],[3].

However, to achieve the performance needed for many engineering applications, the pure PLA is generally reinforced by different type of fibers or fillers. Recentely growing interest in the use of natural/bio-fibers as reinforcements or fillers for biodegradable polymers is noticed, thus creating a new class of materials known as fully "biobased composites" [4].

These natural fibers present many advantages compared to synthetic ones, such as natural availability, low cost, low density, good mechanical properties, eco-friendly and bio-degradability characteristics [5],[6]. They have even the functional capability to substitute or to compete glass fibers by having a higher specific strength and a similar specific[u1] modulus [5],[7]. The most common natural plants used in applications are bast fibers, such as hemp, jute, flax, kenaf, and sisal or agricultural crop residues such as oil palm, pineapple leaf, banana, and sugar palm. They can be obtained in abundance, low cost, and they are also renewable sources of biomass [5],[8]. 
Several research papers deal with the optimization of natural and man-made cellulose fibre-reinforced PLA composites. Some investigate biodegradability, others look for ways to improve temperature resistance or the composites' mechanical characteristics [9]. In this study we have investigated the development of an environmental-friendly composite material prepared from polylactic acid (PLA) and cellulose fibers extracted from Mediterranean saltbush (Atriplex halimus) plant. This plant, commonly used as feed for animals, grows in the dry regions of North Africa and belongs to the Chenopodioideae family [10]. The structure, mechanical and thermal properties of the prepared composites were determined by, FTIR spectroscopy, mechanical tensile tests, and thermogravimetric analysis. Biodegradability and permeability were also investigated.

\section{Materials and methods}

\subsection{Materials}

\subsubsection{Matrix}

Poly (lactic acid) (PLA), film grade was supplied by UMET laboratory (University of Lille 1, France). This biopolymer has a number-average molecular weight $\left(M_{n}\right)$ of 94700 $\mathrm{g} / \mathrm{mol}$, weight-average molecular weight $\left(\mathrm{M}_{\mathrm{w}}\right)$ of $179600 \mathrm{~g} / \mathrm{mol}$ (determined by SEC analysis) and a density of $1.24 \mathrm{~g} / \mathrm{cm}^{3}$.

\subsubsection{Reinforcement}

Cellulose microfibers were extracted from a saltbush (Atriplex halimus) plant (Figure 1), the details of the process have been reported earlier by us [10]. We obtained two sizes of cellulose fibers: short fibers (SF: length $100 \mu \mathrm{m}$, diameter $80 \mathrm{~m}$, aspect ratio 1.25) and long fibers (LF: length $1938 \mu \mathrm{m}$, diameter $430 \mu \mathrm{m}$, aspect ratio 4.5). The average length of the fibers was evaluated by microscopic observation. The chemical composition of the fibers was also estimated after various stages of chemical treatment using standard test methods (ASTM D1103-60 and ASTM D1104-56).

\subsubsection{Chemicals}

Dichloromethane $\left(\mathrm{CH}_{2} \mathrm{Cl}_{2}\right)$ has beenwas used to dissolve PLA and all reagents were supplied by Sigma Aldrich (France).

\subsection{Composites processing}

Solution-cast film samples of the biodegradables materials with $0,5,10$ and $15 \mathrm{wt} \%$ fiber contents were prepared with a dry film thickness of around $100 \mu \mathrm{m}$ and using dichloromethane as a solvent referring to many researchers [11]. $2 \mathrm{~g}$ of PLA was dissolved in $80 \mathrm{ml}$ of dichloromethane $\mathrm{CH}_{2} \mathrm{Cl}_{2}$ and stirred without heating for $30 \mathrm{~min}$ until complete dissolution of PLA, then proper amounts of the cellulose fibers were added and stirred for a further $3 \mathrm{~min}$. subsequentlySubsequently, the mixture was cast onto Petri dishes to generate films after solvent evaporation at room temperature conditions. 
The following terms (PLA) and (PLA-SF5,10,15),(PLA-LF5,10,15) are used to define the non-reinforced PLA film, and PLA composites containing short fibers (SF) and long fibers (LF) $5,10,15 \% \mathrm{wt} / \mathrm{wt}$ of fibers to matrix.

\subsection{Characterization[u2]}

In this work, an Olympus BX41 optical light microscope with a capture camera (Micro Publisher 3.3 RTV) was used to examine the distribution of fibers in the matrix for both sides and to define the dimensions of the cellulose fibers.

Tensile tests were performed on a VERSA Test AFG100N tensile testing machine at a cross-head speed of $14 \mathrm{~mm} / \mathrm{min}$. The test bars had a rectangular cross-section with dimensions of $6 \mathrm{~mm} \times 24 \mathrm{~mm}$ prepared by using a mold and press machine. The data were averaged over five to eight specimens.

Thermogravimetric analysis (TGA) was performed using an ATG PYRIS 1 Perkin Elmer with specimen size of 5 to $10 \mathrm{mg}$. The isothermal tests were carried from 40 to $600^{\circ} \mathrm{C}$ at a heating rate of $20^{\circ} \mathrm{C} / \mathrm{min}$ in the presence of nitrogen.

FT-IR measurements for different films were performed using a Perkin Elmer spectrometer ranging from 4000 to $400 \mathrm{~cm}^{-1}$ to identify different types of chemical bonds existing. The powdered samples and $\mathrm{KBr}$ ( $2 \mathrm{mg}$ sample per $150 \mathrm{mg} \mathrm{KBr}$ ) were pressed into a disk for FT-IR measurement.

Biodegradability of neat PLA and PLA/cellulose composites was evaluated by burying the samples in soil and measuring the weight loss, the soil media used was a mixture of organic hummus and sand. The samples were cut into $30 \times 30 \mathrm{~mm}$ squares, oven dried at $80^{\circ} \mathrm{C}$ to constant weight, weighted for the first time and each sample was placed in vessel containing soil. Exposure to the soil environment continued for 10 months, at predetermined time intervals, samples were taken out from soil, carefully washed with water and dried for weighing and returned again to the soil.

Water absorption of the PLA composites was also determined by placing different samples in an aqueous media. The rate (\%) of absorbed water was calculated by:

$$
\text { Water absorption }(\%)=\frac{m_{f}-m_{i}}{m_{i}} \times 100
$$

where $W_{i}$ is the weight of dried sample before immersion and $W_{f}$ represents the weight of dried material after water immersion.

\section{Results and discussion}

\subsection{Morphology}

The aim of microscopic examination was essentialy to investigate the influence of fiber microstructure on mechanical properties. The micrographs show that generally a 
relatively good dispersion was achieved in composites with low fiber contents even fibers were randomly incorporated.

Figure 2A representing the PLA matrix shows a transparent film with a smooth surface at the exception of the presence of some air bubbles formed after solvent evaporation. The addition of fibers don't do not really affect optical properties of composites such as transparency which remained virtually unmodified compared to the neat PLA [11].

Figure $2 \mathrm{~B}$ and $\mathrm{C}$ shows clear images of the cellulose fibers embedded in the biodegradable matrix, especially short fibers (SF) which were highly more homogeneous than the long fibers (LF). However, a phenomenon of agglomeration of fibers is observed in the formulations containing $15 \%$ of long fibers. This is probably due to the limited amount of polymer which is not sufficient to cover the full surface of the fibers [11],[12].

\subsection{Mechanical analysis[u3]}

Typical stress-strain curves of neat PLA and PLA composites with different fiber contents are shown in Figure 3. A sensitive growing on the tensile properties of composites was noticed with the progressive increments of cellulose fibers.

The modulus of the PLA was increased from 2.4 GPa to $3.1 \mathrm{GPa}$ and $3.5 \mathrm{GPa}$ with the addition of $15 \mathrm{wt} \%$ of short fibers and long fibers respectively, which corresponds to a $26 \%$ and $45 \%$ increase. Similarly for tensile strength, an increase of $40 \%$ and $50 \%$ was observed for PLA-LF15 and PLA-SF15 composites compared to pure PLA. In contrast, a sensitive decrease in the elongation at break from $15 \%$ to $10 \%$ for SF and to $6 \%$ for LF was noticed. These results correspond with many studies where generally we notice an improvement in mechanical properties of composites reinforced by cellulosic fibers or microcrystaline cellulose [13],[14] ],[15],[16].

Higher tensile elastic modulus refers to the rigid property of material that requires more stress to produce the elastic deformation when tensile force is applied [17]. The increase of elastic modulus could be attributed to strong intermolecular force between cellulose fibers and PLA molecules, consequently cellulose fibers particles restricted mobility of PLA polymer chain and resulted in enhancing the stiffness of the composite material [18],[19].

The positive impact of the cellulose fibers essentielly in the composite strength showed that the Atriplex halimus fibers have good mechanical properties and that there are strong interactions between the PLA and the cellulose fibers [12]. However, the large standard deviations reported in Table 1 on the modulus and elongation at break values reported in Table 1 indicated that the fibers, especially long ones, were not homogeneously distributed.

Remarkably, the mechanical response of composites reinforced with SF is markedly superior than to composites with LF essentially due to their small size. This can confirm the proposal of Huda [4], Osswald [20] who suggested that there is a critical fiber length[u4]; if this length is not required the matrix cannot effectively grip the fiber to take the strain, and the fibers will slip and be pulled out, instead of being broken under tension. 
Moreover, Kamonwan [17] indicated that the filler size could have affected the stress distribution between the matrix and filler thus the better mixing or dispersion process of PLA/fibers composites needs to accomplish.

\subsection{Thermal properties}

TGA characterization allows to understand changes in the physical and chemical properties of composites measured as a function of increasing temperature through weight-loss. The results of this test can help to qualify composites for thermally sensitive applications.

The thermal stability of pure PLA and fiber-reinforced PLA composites was investigated with TGA.Thermogravimetric curves of Atriplex halimus fibers, neat PLA and PLA / fibers composites are shown in Figures 6 and 7, respectively.

It is reported that PLA decomposes between 285 and $368^{\circ} \mathrm{C}$, releasing cyclic oligomers, lactide molecules, $\mathrm{CO}$ and $\mathrm{CO}_{2}$ [14],[16]. The TG curve In our case of neat PLA shows a single step thermal degradation at $392^{\circ} \mathrm{C}$.

In case of Atriplex halimus fibers, three-stage weight losses were observed; a first weight loss between $50^{\circ} \mathrm{C}$ and $130^{\circ} \mathrm{C}$ that can be ascribed to moisture loss, Second stage with a small hump in the temperature range from 250 to $300^{\circ} \mathrm{C}$ is characteristic of low molecular weight components, such as hemicelluloses. An other weight loss stage appearing at $358^{\circ} \mathrm{C}$, is corresponded to the thermal degradation of cellulose and Final weight loss stage above $400^{\circ} \mathrm{C}$ is probably due to lignin decomposition suggested by other researchers at $450^{\circ} \mathrm{C}[16],[21]$.

For PLA/ fiber composites, the incorporation of Atriplex halimus fibers in PLA matrix has affected the thermal degradation temperature. The different composites showed a lower degradation temperature (less than $45^{\circ} \mathrm{C}$ ) than that of PLA. Two-stage loss of mass was mainly observed for all composites; in the range from of $300^{\circ} \mathrm{C}$ to $400^{\circ} \mathrm{C}$ due to PLA depolymerization and a second at $350^{\circ} \mathrm{C}$ corresponding to the degradation of cellulose fibers. The thermal stability of composites decreased, which indicates that the distribution of fibers into PLA matrix is not regular with some agglomerations.

We can say that whatever the rate or the type of fiber, the maximum degradation temperatures of the composites is still around $365^{\circ} \mathrm{C}$. Compared to the temperatures recorded by other studies varying between 320 à and $340^{\circ} \mathrm{C}$, these values are very acceptable.

These lower degradation temperatures may be attributed to the decrease of molecular weight of PLA and sometimes to the low of affinity between the fibers/matrix [22],[23],[24].

\subsection{FT-IR analysis}


Typical FT-IR spectra of the PLA and natural fiber reinforced PLA composites are presented in Figure 8. The spectrum of Atriplex halimus fibers is also represented for comparison.

The principal absorbance peaks obtained for the PLA are as follows: the peak around $3512 \mathrm{~cm}^{-1}$ was attributed to the hydroxyl $(\mathrm{OH})$ stretching, the strong peaks at 2996 and 2943 were assigned to the asymmetric and symmetric mode of $\mathrm{C}-\mathrm{H}$ stretching, respectively. The $\mathrm{C}=\mathrm{O}$ stretching (from ester linkage) was observed at 1760 $\mathrm{cm}^{-1}$, the peak at $1457 \mathrm{~cm}^{-1}$ was characterised by the $\mathrm{CH}_{3}$ band, the $\mathrm{C}-\mathrm{H}$ deformation appeared at $1383 \mathrm{~cm}^{-1}$. the O-C asymmetric mode of the ester groups was observed at $1088 \mathrm{~cm}^{-1}$, and the peaks appeared at 957 and $913 \mathrm{~cm}^{-1}$ were attributed to the rocking mode of $\mathrm{CH}_{3}$. Also the peaks at 864 and $753 \mathrm{~cm}^{-1}$, can be referred to the amorphous and crystalline phases of the PLA, respectively [2],[24].

The spectral bands for Atriplex halimus fibers are at $3403 \mathrm{~cm}^{-1}(-\mathrm{OH}$ intermolecular hydrogen bonds) related tot he presence of cellulose and hemicellulose, $2929 \mathrm{~cm}^{-1}(\mathrm{C}-\mathrm{H}$ stretching) present in hemicellulose composent, $1639 \mathrm{~cm}^{-1}$ (frequency of deformation vibration of $\mathrm{CH}_{2}$ and $\mathrm{CH}$ groups are located), $1371 \mathrm{~cm}^{-1}(\mathrm{C}-\mathrm{H}$ band $), 1318 \mathrm{~cm}^{-1}(\mathrm{CH}$ bending mode), $1281 \mathrm{~cm}^{-1}$ (C-O stretching mode of ester group) stretch of the acetyl group in lignins, $1110 \mathrm{~cm}^{-1}$ (C-O-C ether band), and $1051 \mathrm{~cm}^{-1}$ (C-O-C stretching vibration). Previous studies have confirme these trends the spectralof cellulosic materials [14],[16],[17] .

The spectra of the fiber reinforced PLA composites have similar features to those of Pure PLA except for some changes due to the interaction with cellulose fibers; the $\mathrm{OH}$ stretching in the PLA/Fibers composite that appeared at $3437 \mathrm{~cm}^{-1}$, was found to be different to that of the fibres $\left(3496 \mathrm{~cm}^{-1}\right)$ and the PLA $\left(3512 \mathrm{~cm}^{-1}\right)$. This indicates that in the PLA/Fibers composites, the $\mathrm{OH}$ groups (from fibres) formed hydrogen bonds with the carbonyl groups $(\mathrm{C}=0)$ of PLA as shown in Figure 8 . A similar observation has also been reported for the PLA / rice starch composites by other researchers [25],[26]. In addition, it was also evident that the intensity of $C=O\left(1756 \mathrm{~cm}^{-1}\right)$ stretching in the PLA / Fibers composites was significantly higher than that of the PLA.

This was due to the esterification between $\mathrm{OH}$ groups of Atriplex fibres and terminal carboxylic acid groups $(\mathrm{COOH})$ of PLA. This agrees with the work reported by Semba et al [27] who showed that the peak intensity of the $\mathrm{C}=\mathrm{O}$ group increased due to the chemical reaction between the dicumyl peroxide and the PLA in a blend of $\mathrm{PLA} /$ polycaprolactone.

\subsection{Water absorption}

Figure 9 shows the percentage moisture absorption of the pure PLA and PLA/ fibers composites as a function of time of immersion at room temperature. The curve indicates that the water uptake behaviour of PLA composites obeys Fick's law [28], where A rapid absorption was observed within the first days of immersion then slows down to reach the equilibrium after 35 days of immersion.

Water absorption is generally attributed to several factors; firstly, to the presence of some microgaps or defects between the polymer chains. ; sSecondly, to the capillary 
processes through the gaps and flaws at the interface between cellulosic fibers and the PLA matrix. ; Thirdly thirdly to micro-cracks in the matrix formed during processing [16].

In our case this rapid water absorption[u5] at the beginning as water molecules penetrates into the fibers structure, mainly into capillaries as well as spaces between the fibers particles and the matrix.

The addition of long and short cellulose fibers increased the amount of absorbed water; from $0.9 \%$ for neat PLA and $1.6 \%$ for composites containing $15 \mathrm{wt} \%$ of LF. This can be attributed to the hydrophilic nature of the cellulose fibers and to the multiple hydroxyl groups which are available for interaction with the water molecules [25], [29].

\subsection{Biodegradability}

The degradation of PLA is generally made by hydrolysis process which depends essentially on the permeability, the compostion of the polymer and the microbial activity of soil burial. As shown in Figure 9 the samples exhibited a slow weight loss in the first 20 days of soil burial, this period represents the time required for the hydrolysis step and which is confirmed by the water absorption results.

After 3 months of incubtaion incubation many changes in color and shape were observed, than then the sample become became fragile and brittle at the end of six months. After 9 months the they were already in pieces such that only a few parts could be handled. We can say that the necessary period for total degradtion of PLA by soil burial is estimated at 10 months [24], [39].

PLA/fibers showed a higher weight loss than pure PLA with a rate of $92 \%$ and 90 $\%$ for composites containing $15 \mathrm{wt} \%$ of LF and SF due the fact the cellulose fibers are more hydrophilic than the PLA, which accelerates the hydrolysis process. Similar resulst results were reported for PVA/coconut-shell-powder composites [30].

\section{Conclusions}

The incorporation of cellulose fibres in a polymeric matrix is an interesting alternative to develop new materials with good mechanical properties and weak environmental impact. In this context we have investigated the use of new cellulose fibers extracted from Atriplex halimus plant as filler for PLA matrix.

The visual and morphological study of PLA composites showed that a relatively good dispersion was achieved except for somme aggregates in high content of longue fibers. That is why the short fibers were more effective than the long ones by giving better mechanical properties; with a fiber content of $15 \%$ wt of short fibers the tensile strength and the young Young's modulus were raised by $25 \%$ and $45 \%$, respectilvellyrespectively, while the elongation at break was sensibly reduced.

FTIR spectroscopy evidenced the changes in the structure of PLA by adding cellulose fibers and indicated the existence of interaction between $(\mathrm{OH})$ groups of fibers and carboxilic group $(\mathrm{COOH})$ present in PLA.

Furthermore, the addition of the fibers have has weakened the thermal stability and the water resistance of pure PLA by the fact that the cellulose fibers are more hydrophilic. 
Biodegradation tests showed that the incorporation of fibers accelerate the kinetics of degradation, but the most suitable method for PLA composites at the end of thier their cycle of life is composting.

Finally, the prepared composites have potential for considerable growth with a green concept. Primary applications for these biocomposites include, for example, toys for children, furniture, hardware for electronic products, or one-way disposable products, and so on.

Acknowledgements: The authors would like to thank UMET Laboratory of the University Lille 1 (France) for providing the necessary materials and for technical support.

\section{References}

[1] Zhang W, Yang X, Li C, Liang M, Lu C, Deng Y. Mechanochemical activation of cellulose and its thermoplastic polyvinyl alcohol ecocomposites with enhanced physicochemical properties.Carbohydr Polym 2011,83:257-263.

[2] Hapuarachchi TD, Peijs T. Multiwalled carbon nanotubes and sepiolite nanoclays as flame retardants for polylactide and its natural fibre reinforced composites. Composites: Part A 2010,41:954-963.

[3] Frone AN, Berlioz S, Chailan JF, Panaitescu DM. Morphology and thermal properties of PLA-cellulose nanofibers composites. Carbohydr Polym 2013, 91:377-384.

[4] Huda MS, Drzal LT, Mohanty AK, Misra M. Chopped glass and recycled newspaper as reinforcement fibers in injection molded poly(lactic acid) (PLA) composites: A comparative study. Composites Science and Technology 2006, (66)11-12:18131824

[5] Akil HM, Mazuki AAM, Safiee S, Ishak ZAM, Abu Bakar A. Kenaf fiber reinforced composites: A review. Materials and Design 2011, 32:4107-4121.

[6] Frone AN, Berlioz S, Chailan JF, Panaitescu DM. Morphology and thermal properties of PLA-cellulose nanofibers composites. Carbohydrate Polymers 2013 , $91: 377-384$

[7] Bledzki A, Gassan J. Composites reinforced with cellulose based fibers. Prog Polym Sci 1999, 24:221-74

[8] Sahari J, Sapuan SM. Natural fibre reinforced biodegradable polymer composites, Rev.Adv. Mater. Sci 2011, 30: 166-174. 
[9] Nina Graupner N, Axel S. Herrmann AS, Müssig J. Natural and man-made cellulose fibre-reinforced poly(lactic acid) (PLA) composites: An overview about mechanical characteristics and application areas. Composites Part A Applied Science and Manufacturing 2009, 40(6):810-821

[10] Boudjema HL, Bendaikha $\mathrm{H}$. Composite material derived from biodegradable starch polymer and Atriplex Halimus fibers. e-Polymers 2015, 15(6): 419-426.

[11] Sanchez-Garcia MD, Gimenez E, Lagaron JM. Morphology and barrier properties of solvent cast composites of thermoplastic biopolymers and purified cellulose fibers.Carbohydrate Polymers 2008, 71: 235-244.

[12] Nakagaito AN, Fujimura A, Sakai T, Yoshiaki H, Yano H. Production of microfibrillated cellulose (MFC)-reinforced polylactic acid (PLA) nanocomposites from sheets obtained by a papermaking-like process. Composites Science and Technology 2009, 69: 1293-1297.

[13] Jonoobi M, Harun J, Mathew AP, Oksman K. Mechanical properties of cellulose nanofiber (CNF) reinforced polylactic acid (PLA) prepared by twin screw extrusion. Composites Science and Technology 2010, 70: 1742-1747.

[14] Bajwa DS, Adhikari S, Shojaeiarani J, Sreekala G B, Pandey P, Shanmugam SR. Characterization of bio-carbon and ligno-cellulosic fiber reinforced bio-composites with compatibilizer. Construction and Building Materials 2019, 204: 193-202.

[15] Suaduang N, Ross S, Ross G. M, Pratumshat S, Mahasaranon S. Effect of spent coffee grounds filler on the physical and mechanical properties of poly(lactic acid) bio-composite films. Materials Today: Proceedings 2019 17:2104-2110.

[16] Ait Laaziz S, Raji M, Hilali E, Essabir H, Rodrigue D, Bouhfid R, Qaiss A. Biocomposites based on polylactic acid and argan nut shell: Production and properties. International Journal of Biological Macromolecules 2017 0141-8130(17)30599$8[\mathrm{u} 6]$.

[17] Kamonwan AN, Noipitak M. Effect of carbon-rich biochar on mechanical properties of PLA-biochar composites. Sustainable Chemistry and Pharmacy 2020, 15: 100204.

[18] Anwer M A S, Naguib HE, Celzard A., Fierro V. Comparison of the thermal, dynamic mechanical and morphological properties of PLA-Lignin \& PLA-Tannin particulate green composites. Composites Part B: Engineering 2015, 82: 92-99.

[19] Nizamuddin S, Jadhav A., Qureshi SS, Baloch HA, Siddiqui MTH, Mubarak N M, Griffin G, Madapusi S, Tanksale A., Ahamed MI. Synthesis and characterization of polylactide/rice husk hydrochar composite. Scientific Reports 2019, 9: 5445. 
[20] Osswald TA, Fundamental principles of polymer composites: processing and design. In: Proceedings of the 5th international conference of wood fiber-plastic composites. Madison, Wisconsin, May 26-27, 1999.[u7]

[21] Goriparthi BK, Suman KNS, Rao NM. Effect of fiber surface treatments on mechanical and abrasive wear performance of polylactide/jute. Composites Part A: Applied Science and Manufacturing 2012, 43(10): 1800-1808.

[22] Ragoubi M, George B, Molina S, Bienaimé D, Merlin A, Hiver JM, Dahoun A. Effect of corona discharge treatment on mechanical and thermal properties of composites based on miscanthus fibres and polylactic acid or polypropylene matrix. Composites Part A 2012, 43: 675-685.

[23] Sawpan MA, Pickering KL, Fernyhough A. Effect of fibre treatments on interfacial shear strength of hemp fibre reinforced polylactide and unsaturated polyester composites. Composites Part A 2011, 42: 1189-1196.

[24] Bhiogade Y A, Kannan M, Devanathan S .Degradation kinetics study of poly lactic acid (PLA) based biodegradable green composites. Materials Today: Proceedings 2020, 24: 806-814

[25] Ndazi BS, Karlsson S. Characterization of hydrolytic degradation of polylactic acid/rice hulls composites in water at different temperatures. eXPRESS Polymer Letters 2011, 5(2): 119-131.

[26] Lee SH, Ohkita T, Kitagawa K. Eco-composite from poly (lactic acid) and bamboo fiber. Holzforschung 2004, 58: 529-536.

[27] Semba T, Kitagawa K, Ishiaku US, Hamada $H$. The effect of crosslinking on the mechanical properties of polylactic acid/polycaprolactone blends. Journal of Applied Polymer Science 2006, 101: 1816-25.

[28] Li S, McCarthy S. Further investigations on the hydrolytic degradation of poly(DLlactide). Biomaterials 1999, 20(1): 35-44.

[29] Rudeekit Y, Numnoi J, Tajan M, Chaiwutthinan P, Leejarkpai T. Determining biodegradability of polylactic acid under different environments. Journal of Metals, Materials and Minerals 2008, 18(2): 83-87.

[30] Ramaraj B, Poomalai P. Ecofriendly poly(vinyl alcohol) and coconut shell powder composite films: physico-mechanical, thermal properties, and swelling studies. Journal of Applied Polymer Science 2006, 102: 3862-3867.

((captions))

Figure 1: Atriplex halimus plant and optic micrographs of long fibers (LF)/short fibers (SF). 
Figure 2: Optic micrographs of: (A) pure PLA, (B) PLA-SF10\% biocomposite, (c) PLA-LF10\% biocomposites.

Figure 3: The stress-strain curves for PLA composites (A) containing short fibers and (B) long fibers.

Figure 4: The effect of long fibers content on the mechanical properties of PLA composites. Figure 5: The effect of the short fibers content on the mechanical properties of PLA composites. Figure 6 TGA (a) and DTG (b) scans for PLA composites containing 0, 5, 10, $15 \%$ wt of short fibers.

Figure 7: TGA (a) and DTG (b) scans for PLA composites containing $0,5,10,15 \%$ wt of short fibers.

Figure 8: (a) FT-IR spectra for cellulose fibers, non-reinforced PLA and composites, (b): possible interactions between PLA and cellulose fibers.

Figure 9: Weight loss curves of pure PLA, and PLA composites containing $15 \%$ wt of short and long fibers and water absorption as function of storage time, type and the content of fiber. 